\title{
The Effect of Aging on Pacing Strategies in Short and Long Distance Duathlon
}

\author{
Nikolaidis, Pantelis T ; Villiger, Elias ; Victor Sousa, Caio ; Rosemann, Thomas ; Knechtle, Beat
}

\begin{abstract}
Background/Study context: Many studies have been conducted on the triathlon during the last several years; however, less information exists with regards to duathlon (i.e., Run 1, Bike and Run 2). The aim of the present study was to examine the effect of age on pacing (i.e., the relative contribution - \% - of each discipline and transition times - Transition 1 and Transition 2 - to overall race time) of duathletes competing either to short (i.e., $10 \mathrm{~km}$ Run 1, $50 \mathrm{~km}$ Bike and $5 \mathrm{~km}$ Run 2) or long distance (i.e., $10 \mathrm{~km}$ Run 1, $150 \mathrm{~km}$ Bike and $30 \mathrm{~km}$ Run 2). METHODS We analyzed 6,671 finishes (women's, $n=1,037$, age $36.6 \pm 9.1$ years; men's, $n=5,634,40.0 \pm$ 10.0 years) of 3,881 duathletes competing in 'Powerman Zofingen', the World Championship, from 2003 to 2017 , in both the short and the long distance race. RESULTS A large discipline $\times$ distance interaction on relative time (\%) was observed in the short distance $(\mathrm{p}<.001, \eta=.936) ; 24.7 \%, 57.4 \%$, and $15.8 \%$ were spent in Run 1 , Bike, and Run 2, respectively. In the long distance, the relative contribution of disciplines was $8.0 \%, 59.0 \%$, and $32.1 \%$, respectively. A trivial discipline $\times$ sex interaction on relative time $(\%)$ was shown in the short $(p<.001, \eta=.007)$ and long distance $(\mathrm{p}<.001, \eta=.016)$. In the short distance, a small discipline $\times$ age group interaction on relative time (\%) was found ( $\mathrm{p}<.001, \eta=.030)$ with younger age groups spending less time (\%) in Run 1 , Transition 1 and Transition 2, and older groups less time (\%) in Bike and Run 2. In the long distance, a moderate discipline $\times$ age group interaction on relative time $(\%)$ was observed $(\mathrm{p}<.001, \eta=.077)$ with younger age groups spending less time (\%) in Run 1, Transition 1, Transition 2 and Run 2, and older groups less time (\%) in Bike. CONCLUSIONS These findings suggest that younger duathletes are relatively faster in Run 1 and transitions, and older duathletes in Bike in both distances. However, older duathletes are relatively faster in Run 2 in the short distance and younger duathletes are relatively faster in Run 2 in the long distance. The magnitude of the combined effect of discipline and age group on pacing was larger in the long than in the short distance. Therefore, athletes and coaches should be aware of the variation of pacing by age group and distance of a duathlon race such as 'Powerman Zofingen'.
\end{abstract}

DOI: https://doi.org/10.1080/0361073X.2019.1609167

Posted at the Zurich Open Repository and Archive, University of Zurich

ZORA URL: https://doi.org/10.5167/uzh-171208

Journal Article

Accepted Version

Originally published at:

Nikolaidis, Pantelis T; Villiger, Elias; Victor Sousa, Caio; Rosemann, Thomas; Knechtle, Beat (2019). The Effect of Aging on Pacing Strategies in Short and Long Distance Duathlon. Experimental Aging Research, 45(3):223-233. DOI: https://doi.org/10.1080/0361073X.2019.1609167 
3

\title{
The effect of aging on pacing strategies in short and long distance duathlon
}

\author{
Running head: Aging and pacing in duathlon
}

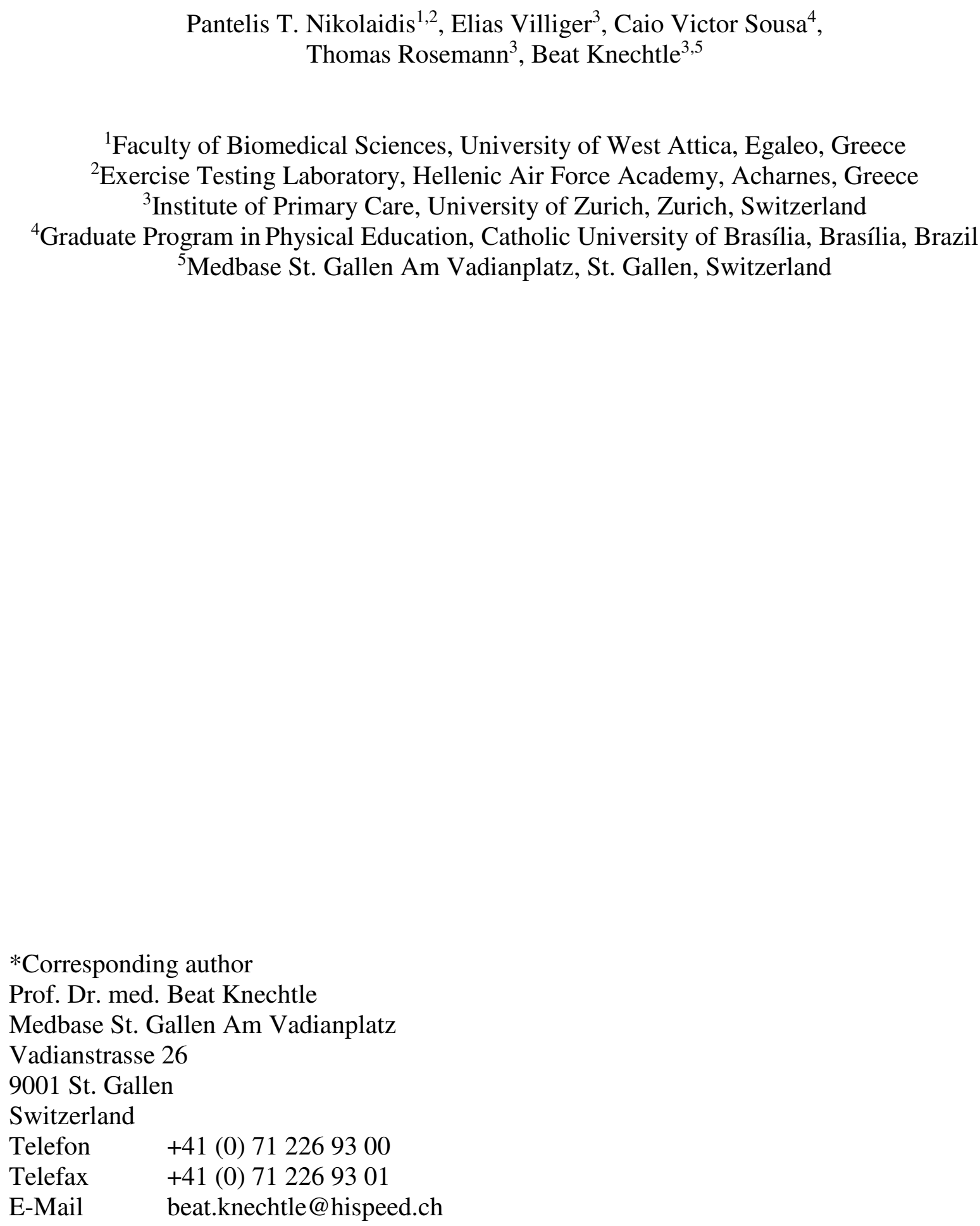


Background/Study context: Many studies have been conducted on the triathlon during the last several years; however, less information exists with regards to duathlon (i.e., Run 1, Bike and Run 2). The aim of the present study was to examine the effect of age on pacing (i.e., the relative contribution - \% - of each discipline and transition times - Transition 1 and Transition 2 - to overall race time) of duathletes competing either to short (i.e., 10km Run 1, 50km Bike and 5km Run 2) or long distance (i.e., 10km Run 1, 150km Bike and 30km Run 2).

Methods: We analyzed 6,671 finishes (women's, $n=1,037$, age 36.6 \pm 9.1 years; men's, $n=5,634,40.0 \pm 10.0$ years) of 3,881 duathletes competing in 'Powerman Zofingen', the World Championship, from 2003 to 2017, in both the short and the long distance race.

Results: A large disciplinexdistance interaction on relative time (\%) was observed in the short distance $\left(\mathrm{p}<.001, \eta_{\mathrm{p}}^{2}=.936\right) ; 24.7 \%, 57.4 \%$ and $15.8 \%$ was spent in Run 1, Bike and Run 2, respectively. In the long distance, the relative contribution of disciplines was $8.0 \%$, $59.0 \%$ and $32.1 \%$, respectively. A trivial disciplinexsex interaction on relative time (\%) was shown in the short $\left(\mathrm{p}<.001, \eta^{2}=.007\right)$ and long distance $\left(p<.001, \eta^{2}=.016\right)$. In the short distance, a small disciplinexage group interaction on relative time $(\%)$ was found $(\mathrm{p}<.001$, $\left.\eta_{p}^{2}=.030\right)$ with younger age groups spending less time (\%) in Run 1, Transition 1 and Transition 2, and older groups less time (\%) in Bike and Run 2. In the long distance, a moderate disciplinexage group interaction on relative time $(\%)$ was observed $(\mathrm{p}<.001$, $\eta_{\mathrm{p}}^{2}=.077$ ) with younger age groups spending less time (\%) in Run 1, Transition 1, Transition 2 and Run 2, and older groups less time (\%) in Bike.

67 Conclusions: These findings suggest that younger duathletes are relatively faster in Run 1 and transitions, and older duathletes in Bike in both distances. However, older duathletes are relatively faster in Run 2 in the short distance and younger duathletes are relatively faster in 
70 Run 2 in the long distance. The magnitude of the combined effect of discipline and age group 71 on pacing was larger in the long than in the short distance. Therefore, athletes and coaches

72 should be aware of the variation of pacing by age group and distance of a duathlon race such 73 as 'Powerman Zofingen'.

74

75 Keywords: aging, cycling, master athletes, running, ultra-endurance 76 


\section{Introduction}

Aging is a natural biological process characterized by cell deterioration and consequently tissue function loss (Sousa-Victor, Garcia-Prat, Serrano, Perdiguero, \& Munoz-Canoves, 2015) that can affect physical performance differently depending on the sport. For instance, athletes running in sprint events reach the peak of performance between 20 to 25 years old, whereas in endurance events, the peak of performance is after 25 years old (Allen \& Hopkins, 2015; Berthelot et al., 2012). In addition to sport performance, pacing - which is a correlate of performance (Abbiss \& Laursen, 2008) has been shown to be influenced by aging in various endurance sports (e.g. marathon running, cross-country skiing), with older athletes presenting a more even pacing than their younger counterparts (Nikolaidis et al., 2018; Nikolaidis \& Knechtle, 2018).

89

Pacing in endurance performance describes how an athlete distributes work and energy throughout an exercise task and the strategy chosen for how an athlete paces the components of a race can have a significant impact on performance (Abbiss \& Laursen, 2008). To date, six different pacing strategies have been identified such as negative, all-out, positive, even, parabolic-shaped and variable pacing strategies (Abbiss \& Laursen, 2008). In the context of a race, a negative pacing occurs when the athlete increases speed across race, whereas a positive pacing refers to the opposite trend.

99 In multi-sports disciplines such as the triathlon (i.e., swimming, cycling, and running), the pacing of athletes has been investigated for different distances such as the sprint distance triathlon (Taylor \& Smith, 2014; Wu et al., 2015; Wu et al., 2016), Olympic 
102 distance, half-Ironman (Stones \& Hartin, 2017; Wu et al., 2015), Ironman triathlon

103 (Angehrn, Rüst, Nikolaidis, Rosemann, \& Knechtle, 2016; Johnson et al., 2015), and

104 longer triathlon distances (Herbst et al., 2011; Knechtle \& Nikolaidis, 2016). In

105 contrast to triathlon, where pacing has been well studied, we have no knowledge of

106 how athletes pace during their races in duathlon (i.e. running, cycling, and running).

107 An important aspect in pacing is the age of the athletes (Nikolaidis \& Knechtle,

108 2018). Since age is related to morphological and physiological adaptations that could

109 affect the energy contribution during a race (Berthelot et al., 2012) the pacing strategy

110 for a young and an older athlete with similar goals could be different. For instance,

111 recent studies in marathoners found that fast master runners (i.e., runners older than

11235 years) pace differently than slow master runners (Nikolaidis \& Knechtle, 2017a).

113 Also, older runners with a similar race time as younger runners pace differently with

114 smaller changes during the race (Nikolaidis \& Knechtle, 2017b). Different from a

115 triathlon, duathlon includes a running leg prior to the cycling and a second running

116 leg after the cycling. This difference between triathlon and duathlon can dramatically

117 change pace strategy to achieve an optimal performance. However, there is no

118 evidence to illustrate the effect of age on pacing strategies used in the duathlon.

119 Therefore, the aim of the present study was to investigate the effect of age on pacing

120 in duathletes and to compare between short- and long-distance duathletes. Based upon

121 previous findings for marathon runners we hypothesize that younger athletes will

122 adopt a more aggressive (i.e., less even) pacing strategy and older athletes maintain a

123 more stable and conservative (i.e., more even) pace throughout the race.

124 


\section{Materials and methods}

126

127

128

129

130

131

132

133

134 We analyzed 6,671 finishes (women's, $n=1,037$, age 36.6 \pm 9.1 years; men's, $n=5,634$, $13540.0 \pm 10.0$ years $)$ of 3,881 duathletes. Most duathletes $(n=2,576)$ had a single finish, 136 whereas 1,305 duathletes had two to 13 finishes. All analyzes were conducted on data 137 derived from the 'Powerman Zofingen' and the 'ITU Powerman Long Distance 138 Duathlon World Championships'. We examined, the effect of age on pacing (i.e., the 139 relative contribution - \% - of each discipline and transition times - Transition 1 and 140 Transition 2 - to overall race time) of duathletes competing either to short (i.e., $10 \mathrm{~km}$ 141 Run 1, 50 km Bike and 5 km Run 2) or long distance (i.e., 10 km Run 1, 150 km Bike 142 and $30 \mathrm{~km}$ Run 2). Transition 1 and Transition 2 refer to the time spent from the end 143 of Run 1 till the start of Bike, and from the end of Bike till the start of Run 2, 144 respectively, and include mainly the change of clothing and/or equipment. The 'Powerman Zofingen' is a duathlon event held in Zofingen (Switzerland) within

\section{Ethical approval}

The Institutional Review Board of Kanton St. Gallen, Switzerland approved all procedures used in the study with a waiver of the requirement for informed consent of the participants given the fact that the study involved the analysis of publicly available data. The study was conducted in accordance with recognized ethical standards of the Declaration of Helsinki adopted in 1964 and revised in 2013. 
150 Powerman World Championships under the name of 'ITU Powerman Long Distance

151 Duathlon World Championships'. The number of participants in this race is not

152 limited and there is not any qualification criterion.

153 A duathlon consists of a running part, a cycling part and then again a running part,

154 which are carried out in immediate sequence. Since 2002, the long distance race of

155 'Powerman Zofingen' has the sequence of $10 \mathrm{~km}$ running, $150 \mathrm{~km}$ cycling and $30 \mathrm{~km}$

156 running. At the same time of the long distance race, there is a short distance race that

157 includes the sequence of $10 \mathrm{~km}$ running, $50 \mathrm{~km}$ cycling and $5 \mathrm{~km}$ running. Before

1582003 , the race course and the distances of the disciplines changed several times since

159 the first edition of the race in 1989. Thus, we analyzed all races from 2003 to 2017 ,

160 i.e. period when there were the same distances for disciplines.

\section{Methodology}

163 Data were obtained from the official race website from 'Powerman Zofingen'

164 www.powerman.ch/de. Race results were sorted by name, age and sex of the finishers

165 separately for both the short and the long distance race. Athletes were ranked in five-

166 year age groups from 15-19 years to 70-74 years and their distribution by sex, age and

167 distance can be seen in Table 1. Performance (i.e., relative time) in each discipline

168 (i.e., Run 1, Bike and Run 2) and transition (i.e., Transition 1 and Transition 2) was

169 expressed as a percentage of the total race time using the formula '100xdisciplines'

170 time/total time'. We studied relative times of disciplines and transitions instead of

171 actual times, since we considered two duathlons differing for distance and,

172 consequently, for total race times.

173 


\section{Statistical analysis}

175 Data are presented as means \pm standard deviations. A between-within measures

176 analysis of variance (ANOVA) examined the distancexdiscipline interaction on

177 relative time, i.e. whether the percentage contribution of each discipline to the total

178 race time varied by race distance. Within each distance, a between-within measures

179 ANOVA examined the sex $\times$ discipline and age group $\times$ discipline interaction on

180 relative time. A one-way ANOVA examined the main effect of age group on relative

181 time for each discipline separately. The magnitude of these interactions was examined

182 using effect size partial eta square $\left(\eta^{2}\right)$ and was evaluated as following: small $(0.010$

$\left.183<\eta_{p}^{2} \leq 0.059\right)$, moderate $\left(0.059<\eta^{2} \leq 0.138\right)$ and large $\left(\eta_{p}^{2}>0.138\right)$ effect estimates

184 (Cohen, 1988). In addition, to analyze the main effects of sex and age group, and their

185 interaction on relative time spent in each discipline and transition (\%), a mixed-effects

186 regression model with each finisher as a random variable was used to consider

187 finishers who completed several races. We included 'sex' and 'age group' as fixed

188 variables in our analysis. Statistical analyses were carried out using GraphPad Prism

189 v. 7.0 (GraphPad Software, San Diego, USA) and IBM SPSS v.23.0 (SPSS, Chicago,

190 USA). 


\section{Results}

192

193 Performance in total and in each discipline (i.e., Run 1, Bike and Run 2) can be seen in Table 2. A large disciplinexdistance interaction on relative time (\%) was observed

195

212 In the short distance race, time $(\%)$ differed among age groups for Run $1\left(\mathrm{~F}_{(11,3489)}=\right.$ $\left.2139.826, \mathrm{p}<.001, \eta_{\mathrm{p}}^{2}=.030\right)$, Transition $1\left(\mathrm{~F}_{(11,3489)}=30.009, \mathrm{p}<.001, \eta_{\mathrm{p}}^{2}=.086\right)$, 214 Bike $\left(\mathrm{F}_{(11,3489)}=12.186, \mathrm{p}<.001, \eta^{2} \mathrm{p}=.037\right)$, Transition $2\left(\mathrm{~F}_{(11,3489)}=36.650, \mathrm{p}<\right.$ 215 in the short distance race $\left(\mathrm{F}_{(1.425,9517.786)}=98000.346, \mathrm{p}<.001, \eta_{\mathrm{p}}^{2}=.936\right) ; 24.7 \%$, $57.4 \%$ and $15.8 \%$ was spent in Run1, Bike and Run2, respectively. In the long distance race, the relative contribution of disciplines was $8.0 \%, 59.0 \%$ and $32.1 \%$, respectively. A trivial-to-small disciplinexsex interaction on relative time (\%) was shown in the short distance run $\left(\mathrm{F}_{(1.611,5644.023)}=25.093, \mathrm{p}<.001, \eta_{\mathrm{p}}^{2}=.007\right)$ and the long distance run $\left(\mathrm{F}_{(1.098,3481.231)}=52.095, \mathrm{p}<.001, \eta_{\mathrm{p}}^{2}=.016\right)$.

In the short distance race, a small disciplinexage group interaction on relative time $(\%)$ was found $\left(\mathrm{F}_{(19.272,5609.842)}=9.402, \mathrm{p}<.001, \eta_{\mathrm{p}}^{2}=.031\right)$ with athletes in younger age groups spending less time (\%) in Run 1, Transition 1 and Transition 2, and athletes in older groups less time (\%) in Bike and Run 2 (Figure 1).

In the long distance race, a moderate disciplinexage group interaction on relative time $(\%)$ was observed $\left(\mathrm{F}_{(13.198,3475.462)}=22.065, \mathrm{p}<.001, \eta_{\mathrm{p}}^{2}=.077\right)$ with athletes in younger age groups spending less time (\%) in Run 1, Transition 1, Transition 2 and Run 2, and athletes in older groups less time (\%) in Bike (Figure 2). $\left..001, \eta_{\mathrm{p}}^{2}=.093\right)$ and Run $2\left(\mathrm{~F}_{(11,3489)}=2.879, \mathrm{p}=.001, \eta_{\mathrm{p}}^{2}=.009\right)$. The younger 
216 athletes spent relatively less time (\%) in Run 1, Transition 1 and Transition 2, whereas

217 the opposite trend was observed in Bike. The magnitude of the age effect ranged from

218 small to moderate, and was the strongest in Transition 2 and the weakest in Run 2.

220 In the long distance race, time $(\%)$ differed among age groups. for Run $1\left(\mathrm{~F}_{(11,3158)}=\right.$

221 12.290, $\left.\mathrm{p}<.001, \eta_{\mathrm{p}}^{2}=.041\right)$, Transition $1\left(\mathrm{~F}_{(11,3158)}=24.362, \mathrm{p}<.001, \eta_{\mathrm{p}}^{2}=.078\right)$,

222 Bike $\left(\mathrm{F}_{(11,3158)}=29.787, \mathrm{p}<.001, \eta_{\mathrm{p}}^{2}=.094\right)$, Transition $2\left(\mathrm{~F}_{(11,3158)}=19.081, \mathrm{p}<\right.$

$\left.223.001, \eta_{\mathrm{p}}^{2}=.062\right)$ and Run $2\left(\mathrm{~F}_{(11,3158)}=18.234, \mathrm{p}<.001, \eta_{\mathrm{p}}^{2}=.060\right)$. The younger

224 athletes spent relatively less time (\%) in Run 1, Run 2, Transition 1 and Transition 2,

225 whereas the opposite trend was shown in Bike. The magnitude of the age effect

226 ranged from small to moderate, and was the strongest in Bike and the weakest in Run

227 1. The results of the mixed-effects regression analysis of relative (\%) and actual times

228 were presented in Table 3 and Table 4, respectively. This analysis highlighted the

229 main effects of sex and age group on relative time (\%) spent in each discipline.

230

231 


\section{Discussion}

234 This study investigated for the first time the effect of age on pacing in athletes

235 competing in two different duathlon distances, short and long, both in the 'Powerman

236 Zofingen' held as the 'ITU Powerman Long Distance Duathlon World

237 Championships'. The most important findings are that $(i)$ younger duathletes are

238 relatively (\%) faster than their older counterparts in the first run and transitions in

239 short distance race, (ii) older duathletes are relatively (\%) faster than younger

240 duathletes in cycling in both the short and the long distance race, (iii) older duathletes

241 are relatively (\%) faster than their younger counterparts in the second run in the short

242 distance race and (iv) younger duathletes are relatively (\%) faster than older

243 duathletes in the second run in the long distance race.

Younger duathletes are relatively faster in the first run and transitions in the short

\section{distance}

247 A first important finding was that younger athletes were relatively faster in the first

248 running discipline in the short distance race. Although young athlete may have an

249 enhanced performance due to physiological causes, this faster first run are probably

250 due to pacing strategy, since older duathletes were faster in cycling and second

251 running leg. Similarly, older marathoners were also faster than younger in the 'New

252 York City Marathon' (Nikolaidis \& Knechtle, 2017b).

254 For the aspect of transition times in multi-sports disciplines, very little is known

255 (Rüst, Rosemann, Lepers, \& Knechtle, 2014). When athletes competing in 'Ironman

256 Hawaii' were compared to athletes competing in 'Ironman 70.3' between 1998 and 
2013, transition times increased for both women and men whereas the sex difference decreased over time. Transition times decreased for both women and men whereas the sex difference remained unchanged in 'Ironman 70.3'. Generally, transition times were slower in 'Ironman Hawaii' compared to 'Ironman 70.3' (Rüst, Rosemann, Lepers, \&

261 Knechtle, 2014).

Younger are relatively faster in the second run in the long distance race

A second important finding was that younger athletes were relatively faster in the second running discipline in the long distance race. The second running leg of long distance duathlon comprises $30-\mathrm{km}$ after $10-\mathrm{km}$ run and $150-\mathrm{km}$ cycling. Although older athletes seem to adopt conservative pacing strategies to endure their maximal capacity during the entire race, it is not enough to go faster than younger duathletes.

269 While aging, the human body undergoes several adaptations that decrease the

270 fundamental factors underlying endurance performance, such as running economy and 271 maximal oxygen uptake (Everman, Farris, Bay, \& Daniels, 2018; Quinn, Manley, 272 Aziz, Padham, \& MacKenzie, 2011).

274 Quinn et al. (2011) evaluated young, master and older endurance runners in a cross275 section design and reported that the main factors affecting performance are maximal 276 and submaximal cardiorespiratory capacity and decreases in strength and power.

277 Many of these detrimental factors to performance may be attenuated in a single race 278 when experienced athletes apply proper pacing strategy, as showed in our short 279 distance results. However, it seems that only proper pacing strategy is not enough to 280 go faster than younger athletes in long distance duathlon. 
283 A third important finding was that older athletes were relatively faster in the cycling 284 discipline in both the short and the long distance race. This finding might be due to 285 the age-related performance decline in cycling which occurs later in life than the age286 related performance decline in running. When the master World records in 1-h track 287 cycling, 1500-m swimming and 10-km running in athletes older than 60 years were analyzed the maintenance of high performance in cycling persists longer into old age than for running or swimming (Lepers, Stapley, \& Cattagni, 2017).

\section{Older are relatively faster in the second run in the short distance race}

292 A fourth important finding was that older athletes were relatively faster in the second run discipline in the shorter race distance. A potential explanation for this finding could be the aspect of experience in pacing with increased age. When marathoners competing in the 'New York City Marathon' between 2006 and 2016 were analyzed, marathoners in older age groups presented a relatively more even pace compared to marathoners in younger age groups (Nikolaidis \& Knechtle, 2017b). Similarly for 100-km ultra-marathoners, athletes in younger age groups (i.e. 18-24 years) were slower than athletes in most other age groups and athletes in older age groups showed no trend of slowing down (Rüst, Rosemann, Zingg, \& Knechtle, 2015). It could also

301 be demonstrated that ultra-marathoners in age group 40-44 years were the best pacers 302 in a 100-km ultra-marathon and were able to achieve a negative pacing in the last 303 segment of the race (Knechtle, Rosemann, Zingg, Stiefel, \& Rüst, 2015). in performance. Duathletes are characterized by high maximal oxygen uptake 
$307\left(\mathrm{VO}_{2 \max }, 67 \mathrm{~mL} \cdot \mathrm{min}^{-1} \cdot \mathrm{kg}^{-1}\right)$ and large-to-very large correlation between $\mathrm{VO}_{2 \max }$ and

308 race time has been observed (Tong \& Rees, 1996). Moreover, aerobic capacity

309 expressed as maximal workload and maximal velocity attained during an incremental

310 exercise test were the best predictors of duathlon performance (Chavarren Cabrero,

311 Jimenez Ramirez, Dorado Garcia, Ballesteros Martinez-Elorza, \& Lopez Calbet,

312 1997). The energy cost of Run 1 and Run 2 are similar (Vallier, Mazure, Hausswirth,

313 Bernard, \& Brisswalter, 2003). A study of heart rate responses to duathlon showed

314 similar scores among the three disciplines (Ronconi \& Alvero-Cruz, 2011). Since

$315 \mathrm{VO}_{2 \max }$ declines with aging it is reasonable to observe corresponding age-related

316 changes in pacing.

318 A limitation of the present study was that the findings were specific for the duathlons

319 with similar characteristics as those of 'Powerman Zofingen' and should be

320 generalized with caution to other duathlon races. Strength of the study was that it

321 filled a gap in the existing literature as no previous study examined the agexpacing

322 interaction in duathlon. During the recent years, an increased number of finishers has

323 been observed in 'Powerman Zofingen' (Rüst et al., 2013); thus, our results might

324 impact on increased numbers of duathletes. Coaches and fitness trainers working with

325 duathletes can use these findings to develop age-tailored pacing strategies. It should

326 be also highlighted that the magnitude of age-related differences in pacing in the short

327 distance was stronger in transitions than in the three disciplines. The different trend

328 was observed in the long distance, where the strongest effect of age was shown in the

329 Bike and the weakest in Run 1. Moreover, since the magnitude of age-related

330 differences was stronger in the three disciplines in the long than in the short distance, 
331 considering the age of duathletes in designing pacing strategy should be more

332 important in long than in short distance.

\section{Conclusion}

335 In summary, younger duathletes are relatively (\%) faster than older duathletes in the

336 first running discipline and in the transition area, whereas older duathletes are

337 relatively faster than their younger counterparts in cycling in both the short and the

338 long distance race. And, older duathletes are relatively faster than younger duathletes

339 in the second run in the short distance race, whereas younger duathletes are relatively

340 faster than older duathletes in the second run in the long distance race. Athletes and

341 coaches should be aware of the variation of pacing by age group and distance of a

342 duathlon race such as 'Powerman Zofingen'. 


\section{References}

345 Abbiss, C. R., \& Laursen, P. B. (2008). Describing and understanding pacing strategies during athletic competition. Sports Medicine, 38(3), 239-252.

Allen, S. V., \& Hopkins, W. G. (2015). Age of peak competitive performance of elite athletes: A systematic review. Sports Medicine, 45(10), 1431-1441. doi: $10.1007 / \mathrm{s} 40279-015-0354-3$

Angehrn, N., Rust, C. A., Nikolaidis, P. T., Rosemann, T., \& Knechtle, B. (2016). Positive pacing in elite Ironman triathletes. Chinese Journal of Physiology, 59(6), 305-314. doi: 10.4077/cjp.2016.bae418

Berthelot, G., Len, S., Hellard, P., Tafflet, M., Guillaume, M., Vollmer, J. C., . . . Toussaint, J. F. (2012). Exponential growth combined with exponential decline explains lifetime performance evolution in individual and human

Chavarren Cabrero, J., Jimenez Ramirez, J., Dorado Garcia, C., Ballesteros MartinezElorza, J. M., \& Lopez Calbet, J. A. (1997). Prediction of performance in duathlon competititon. Archivos de Medicina del Deporte, 14(57), 17-24.

Cohen, J. (1988). Statistical power analysis for the behavioral sciences. Hillsdale, NJ: Lawrence Erlbaum Associates.

362 Everman, S., Farris, J. W., Bay, R. C., \& Daniels, J. T. (2018). Elite distance runners: A 45-year follow-up. Medicine and Science in Sports and Exercise, 50(1), 7378. doi: 10.1249/MSS.0000000000001407

365 Herbst, L., Knechtle, B., Lopez, C. L., Andonie, J. L., Fraire, O. S., Kohler, G., . . Rosemann, T. (2011). Pacing strategy and change in body composition during 
a Deca Iron triathlon. Chinese Journal of Physiology, 54(4), 255-263. doi: 10.4077/cjp.2011.amm115

Johnson, E. C., Pryor, J. L., Casa, D. J., Belval, L. N., Vance, J. S., DeMartini, J. K., . . Armstrong, L. E. (2015). Bike and run pacing on downhill segments predict Ironman triathlon relative success. Journal of Science and Medicine in Sport, 18(1), 82-87. doi: 10.1016/j.jsams.2013.12.001

Knechtle, B., \& Nikolaidis, P. T. (2016). Sex differences in pacing during 'Ultraman Hawaii'. PeerJ, 4, e2509. doi: 10.7717/peerj.2509

Knechtle, B., Rosemann, T., Zingg, M. A., Stiefel, M., \& Rüst, C. A. (2015). Pacing strategy in male elite and age group $100 \mathrm{~km}$ ultra-marathoners. Open Access Journal of Sports Medicine, 6, 71-80. doi: 10.2147/oajsm.s79568

Lepers, R., Stapley, P. J., \& Cattagni, T. (2017). Age-related changes in endurance performance vary between modes of locomotion in men: An analysis of master world records. International Journal of Sports Physiology and Performance, in print. doi: 10.1123/ijspp.2017-0222

Nikolaidis, P. T., \& Knechtle, B. (2017a). Do fast older runners pace differently from 383 fast younger runners in the 'new york city marathon'? Journal of Strength and Conditioning Research, in print. doi: 10.1519/jsc.0000000000002159

Nikolaidis, P. T., \& Knechtle, B. (2017b). Effect of age and performance on pacing of marathon runners. Open Access Journal of Sports Medicine, 8, 171-180. doi:

388 Nikolaidis, P. T., \& Knechtle, B. (2018). Pacing in age group marathoners in the "New York City Marathon". Research in Sports Medicine, 26(1), 86-99. doi: $10.1080 / 15438627.2017 .1393752$ 
391 Nikolaidis, P.T., Villiger, E., Rosemann, T., \& Knechtle, B. (2018). The effect of aging on pacing strategies of cross-country skiers and the role of performance level. European Review of Aging and Physical Activity, 15, 4. doi: 10.1186/s11556-018-0193-y

Quinn, T. J., Manley, M. J., Aziz, J., Padham, J. L., \& MacKenzie, A. M. (2011). Aging and factors related to running economy. Journal of Strength and Conditioning Research, 25(11), 2971-2979. doi: 10.1519/JSC.0b013e318212dd0e

Ronconi, M., \& Alvero-Cruz, J. R. (2011). Heart rate and oxygen uptake responses in male athletes in duathlon sprint competitions. Apunts Medicina de l'Esport, 46(172), 183-188.

402

403

404

405

406

407

408

Rüst, C. A., Knechtle, B., Knechtle, P., Pfeifer, S., Rosemann, T., Lepers, R., \& Senn, O. (2013). Gender difference and age-related changes in performance at the long-distance duathlon. Journal of Strength and Conditioning Research, 27(2), 293-301.

Rüst, C. A., Rosemann, T., Lepers, R., \& Knechtle, B. (2014). Changes in transition times in 'Ironman Hawaii' between 1998 and 2013. BMC Sports Science, Medicine and Rehabilitation, 6, 37. doi: 10.1186/2052-1847-6-37

Rüst, C. A., Rosemann, T., Zingg, M. A., \& Knechtle, B. (2015). Do non-elite older runners slow down more than younger runners in a $100 \mathrm{~km}$ ultra-marathon? BMC Sports Science, Medicine and Rehabilitation, 7, 1. doi: 10.1186/20521847-7-1

Sousa-Victor, P., Garcia-Prat, L., Serrano, A. L., Perdiguero, E., \& Munoz-Canoves, P. (2015). Muscle stem cell aging: Regulation and rejuvenation. Trends in 
Stones, M.J. \& Hartin, A. (2017) Aging and Half-Ironman performance. Experimental Aging Research, 43, 178-191. doi: 10.1080/0361073X.2017.1276378

Taylor, D., \& Smith, M. F. (2014). Effects of deceptive running speed on physiology, perceptual responses, and performance during sprint-distance triathlon. Physiology \& Behavior, 133, 45-52. doi: 10.1016/j.physbeh.2014.05.002

Tong, R. J., \& Rees, J. A. (1996). Determinants of performance in the duathlon. Journal of Sports Sciences, 14(1), 58-59.

Vallier, J. M., Mazure, C., Hausswirth, C., Bernard, T., \& Brisswalter, J. (2003). Energy cost of running in a specified duathlon series. Canadian Journal of Applied Physiology, 28(5), 673-684.

Wu, S. S., Peiffer, J. J., Brisswalter, J., Nosaka, K., Lau, W. Y., \& Abbiss, C. R. (2015). Pacing strategies during the swim, cycle and run disciplines of sprint, Olympic and half-Ironman triathlons. European Journal of Applied Physiology, 115(5), 1147-1154. doi: 10.1007/s00421-014-3096-2

Wu, S. S., Peiffer, J. J., Peeling, P., Brisswalter, J., Lau, W. Y., Nosaka, K., \& Abbiss, C. R. (2016). Improvement of sprint triathlon performance in trained athletes with positive swim pacing. International Journal of Sports Physiology and 
Table 1 Finishes by distance, sex and age group

440

\begin{tabular}{ccccc}
\hline & \multicolumn{2}{c}{ Short distance } & \multicolumn{2}{c}{ Long distance } \\
\hline Age group & Women $(n)$ & Men $(n)$ & Women $(n)$ & Men $(n)$ \\
\hline $15-19$ & 24 & 107 & 1 & 2 \\
$20-24$ & 49 & 133 & 7 & 70 \\
$25-29$ & 100 & 275 & 68 & 271 \\
$30-34$ & 108 & 409 & 103 & 444 \\
$35-39$ & 99 & 521 & 89 & 511 \\
$40-44$ & 89 & 556 & 76 & 541 \\
$45-49$ & 56 & 450 & 76 & 394 \\
$50-54$ & 25 & 248 & 42 & 235 \\
$55-59$ & 6 & 145 & 14 & 124 \\
$60-64$ & 0 & 77 & 4 & 59 \\
$65-69$ & 0 & 22 & 1 & 32 \\
$70-74$ & 0 & 2 & 0 & 6 \\
\hline Total & 556 & 2,945 & 481 & 2,689 \\
\hline
\end{tabular}

441

442

443 
Table 2 Time (min) spent in disciplines and transitions by distance 445

\begin{tabular}{lcc}
\hline & Short distance & Long distance \\
\hline Run 1 & $42.9 \pm 5.8$ & $40.5 \pm 5.2$ \\
Transition 1 & $1.8 \pm 0.7$ & $2.1 \pm 0.9$ \\
Bike & $99.7 \pm 11.8$ & $299.8 \pm 32.1$ \\
Transition 2 & $1.8 \pm 0.7$ & $2.7 \pm 1.2$ \\
Run 2 & $27.6 \pm 4.9$ & $164.1 \pm 26.2$ \\
\hline Total & $174.0 \pm 21.6$ & $509.1 \pm 59.6$ \\
\hline
\end{tabular}

446

447 
Table 3 Mixed-effects regression analysis of relative time (\%) by sex and group

\begin{tabular}{|c|c|c|c|c|c|c|}
\hline \multirow[b]{2}{*}{ Parameter } & \multicolumn{3}{|c|}{ Short distance } & \multicolumn{3}{|c|}{ Long distance } \\
\hline & Estimate & SEE & Significance & Estimate & SEE & Significance \\
\hline \multicolumn{7}{|l|}{ Run 1} \\
\hline Intercept & 24.03 & 0.08 & $<0.001$ & 7.65 & 0.03 & $<0.001$ \\
\hline Age group & 0.12 & 0.01 & $<0.001$ & 0.05 & 0.01 & $<0.001$ \\
\hline Women & 0.68 & 0.19 & $<0.001$ & 0.18 & 0.09 & 0.034 \\
\hline $\begin{array}{l}\text { Age } \\
\text { group*Women }\end{array}$ & -0.13 & 0.04 & 0.001 & \multicolumn{2}{|c|}{ group*Women } & 0.456 \\
\hline Transition 1 & & & & & & \\
\hline Intercept & 0.82 & 0.02 & $<0.001$ & 0.31 & 0.01 & $<0.001$ \\
\hline Age group & 0.04 & $<0.01$ & $<0.001$ & 0.02 & $<0.01$ & $<0.001$ \\
\hline Women & -0.01 & 0.04 & 0.866 & 0.01 & 0.02 & 0.761 \\
\hline $\begin{array}{l}\text { Age } \\
\text { group*Women }\end{array}$ & $<0.01$ & 0.01 & 0.585 & $<0.01$ & $<0.01$ & 0.474 \\
\hline \multicolumn{7}{|l|}{ Bike } \\
\hline Intercept & 58.26 & 0.12 & $<0.001$ & 60.72 & 0.15 & $<0.001$ \\
\hline Age group & -0.17 & 0.02 & $<0.001$ & -0.34 & 0.02 & $<0.001$ \\
\hline Women & 0.11 & 0.29 & 0.715 & 1.25 & 0.39 & 0.001 \\
\hline $\begin{array}{l}\text { Age } \\
\text { group*Women }\end{array}$ & 0.06 & 0.06 & 0.302 & -0.09 & 0.07 & 0.178 \\
\hline \multicolumn{7}{|l|}{ Transition 2} \\
\hline Intercept & 0.82 & 0.02 & $<0.001$ & 0.40 & 0.01 & $<0.001$ \\
\hline Age group & 0.04 & $<0.01$ & $<0.001$ & 0.02 & $<0.01$ & $<0.001$ \\
\hline Women & -0.06 & 0.04 & 0.186 & -0.02 & 0.03 & 0.607 \\
\hline $\begin{array}{l}\text { Age } \\
\text { group*Women }\end{array}$ & 0.01 & 0.01 & 0.161 & $<0.01$ & 0.01 & 0.598 \\
\hline \multicolumn{7}{|l|}{ Run 2} \\
\hline Intercept & 16.11 & 0.08 & $<0.001$ & 30.94 & 0.14 & $<0.001$ \\
\hline Age group & -0.04 & 0.01 & 0.001 & 0.24 & 0.02 & $<0.001$ \\
\hline Women & -0.75 & 0.19 & $<0.001$ & -1.43 & 0.37 & $<0.001$ \\
\hline $\begin{array}{l}\text { Age } \\
\text { group*Women }\end{array}$ & 0.06 & 0.04 & 0.129 & 0.11 & 0.07 & 0.112 \\
\hline
\end{tabular}


Table 4 Mixed-effects regression analysis of actual time by sex and group

\begin{tabular}{|c|c|c|c|c|c|c|}
\hline \multirow[b]{2}{*}{ Parameter } & \multicolumn{3}{|c|}{ Short distance } & \multicolumn{3}{|c|}{ Long distance } \\
\hline & Estimate & SEE & Significance & Estimate & SEE & Significance \\
\hline \multicolumn{7}{|l|}{ Run 1} \\
\hline Intercept & 38.81 & 0.29 & $<0.001$ & 34.79 & 0.27 & $<0.001$ \\
\hline Age group & 0.65 & 0.05 & $<0.001$ & 0.97 & 0.05 & $<0.001$ \\
\hline Women & 6.41 & 0.72 & $<0.001$ & 3.28 & 0.72 & $<0.001$ \\
\hline $\begin{array}{l}\text { Age } \\
\text { group*Women }\end{array}$ & -0.50 & 0.14 & $<0.001$ & 0.16 & 0.13 & 0.203 \\
\hline \multicolumn{7}{|l|}{ Transition 1} \\
\hline Intercept & 1.29 & 0.04 & $<0.001$ & 1.37 & 0.06 & $<0.001$ \\
\hline Age group & 0.10 & 0.01 & $<0.001$ & 0.14 & 0.01 & $<0.001$ \\
\hline Women & 0.18 & 0.09 & $<0.001$ & 0.14 & 0.15 & 0.325 \\
\hline $\begin{array}{l}\text { Age } \\
\text { group*Women }\end{array}$ & $<0.01$ & 0.02 & 0.814 & $<0.01$ & 0.03 & 0.934 \\
\hline \multicolumn{7}{|l|}{ Bike } \\
\hline Intercept & 93.59 & 0.65 & $<0.001$ & 276.64 & 1.86 & $<0.001$ \\
\hline Age group & 0.86 & 0.11 & $<0.001$ & 3.86 & 0.31 & $<0.001$ \\
\hline Women & 12.35 & 1.57 & $<0.001$ & 26.78 & 4.84 & $<0.001$ \\
\hline $\begin{array}{l}\text { Age } \\
\text { group*Women }\end{array}$ & -0.50 & 0.31 & 0.110 & 0.64 & 0.85 & 0.450 \\
\hline \multicolumn{7}{|l|}{ Transition 2} \\
\hline Intercept & 1.29 & 0.04 & $<0.001$ & 1.77 & 0.08 & $<0.001$ \\
\hline Age group & 0.10 & 0.01 & $<0.001$ & 0.17 & 0.01 & $<0.001$ \\
\hline Women & 0.09 & 0.09 & 0.326 & 0.01 & 0.21 & 0.978 \\
\hline $\begin{array}{l}\text { Age } \\
\text { group*Women }\end{array}$ & 0.02 & 0.02 & 0.407 & 0.04 & 0.04 & 0.245 \\
\hline \multicolumn{7}{|l|}{ Run 2} \\
\hline Intercept & 26.07 & 0.29 & $<0.001$ & 139.15 & 1.56 & $<0.001$ \\
\hline Age group & 0.23 & 0.05 & $<0.001$ & 4.55 & 0.26 & $<0.001$ \\
\hline Women & 2.11 & 0.69 & 0.002 & 2.32 & 4.04 & 0.566 \\
\hline $\begin{array}{l}\text { Age } \\
\text { group*Women }\end{array}$ & -0.10 & 0.14 & 0.469 & 1.32 & 0.71 & 0.063 \\
\hline
\end{tabular}




\section{$456 \quad$ List of figures}

457

458

459 Figure 1 Time (\%) in each discipline and transition by age group in short

460

distance

461

462 Figure 2 Time (\%) in each discipline and transition by age group in long

463

distance

464

465

466

467

468

469

470

471

472

473

474

475

476

477

478

479

480

481

482

483

484

485

486

487

488

489

490

491

492

493

494

495

496

497 
Run1

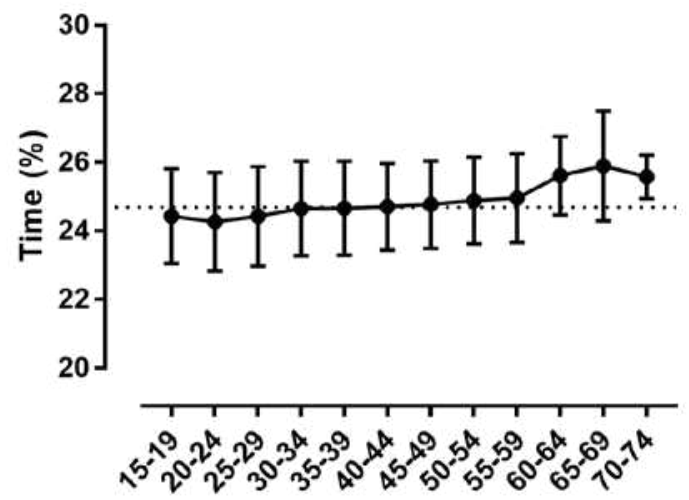

Age group
Bike

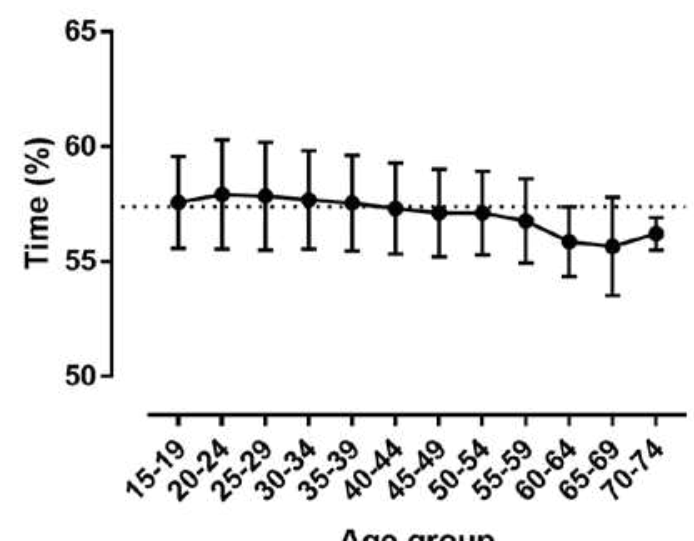

Run2

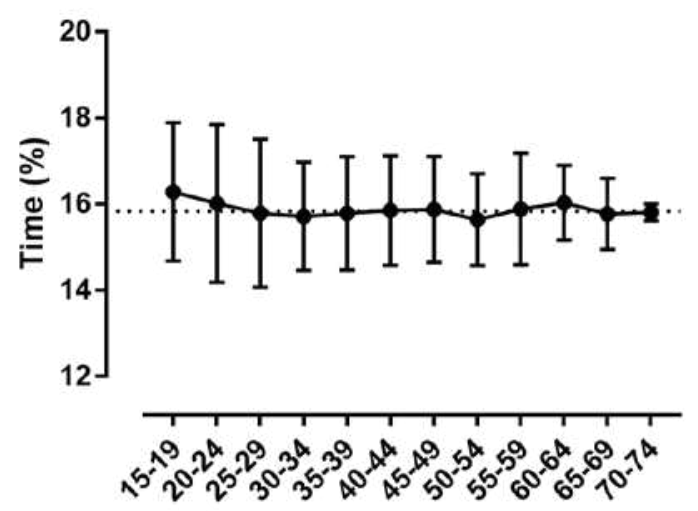

Age group

Transition 1

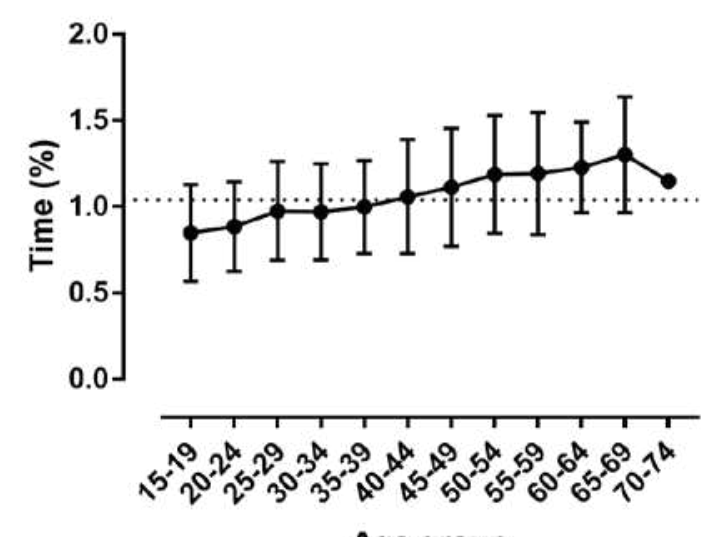

Age group

Transition 2

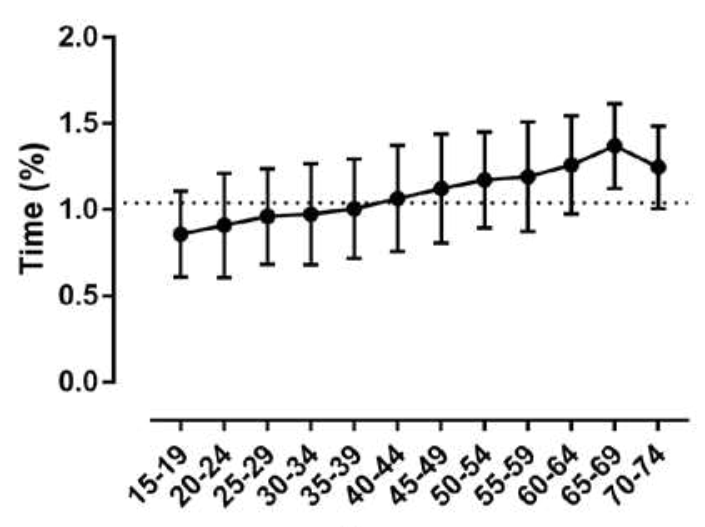

Age group 


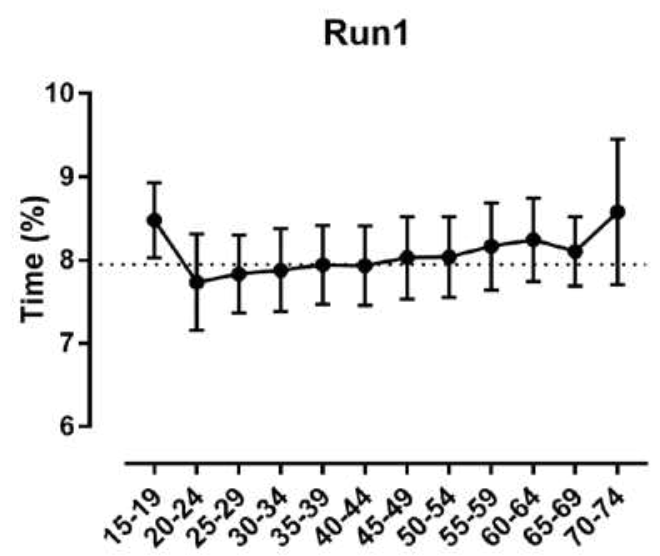

Age group
Bike

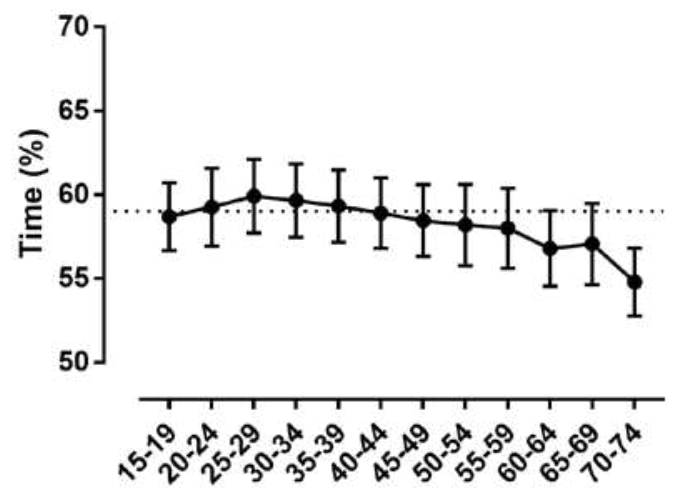

Age group
Run2

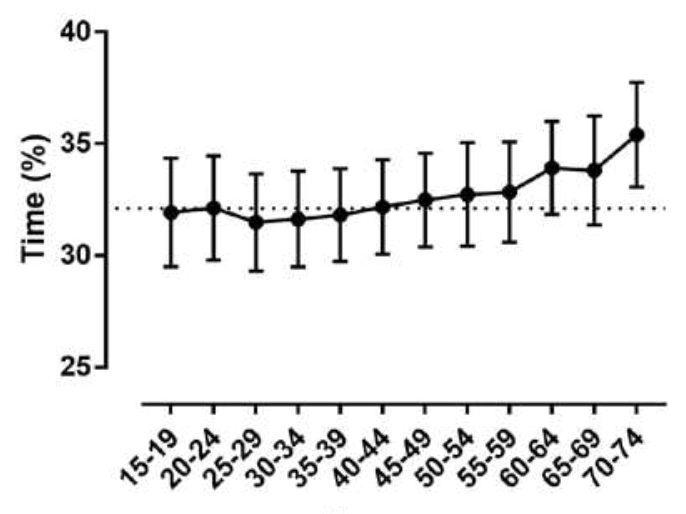

Age group

Transition 1

Transition 2
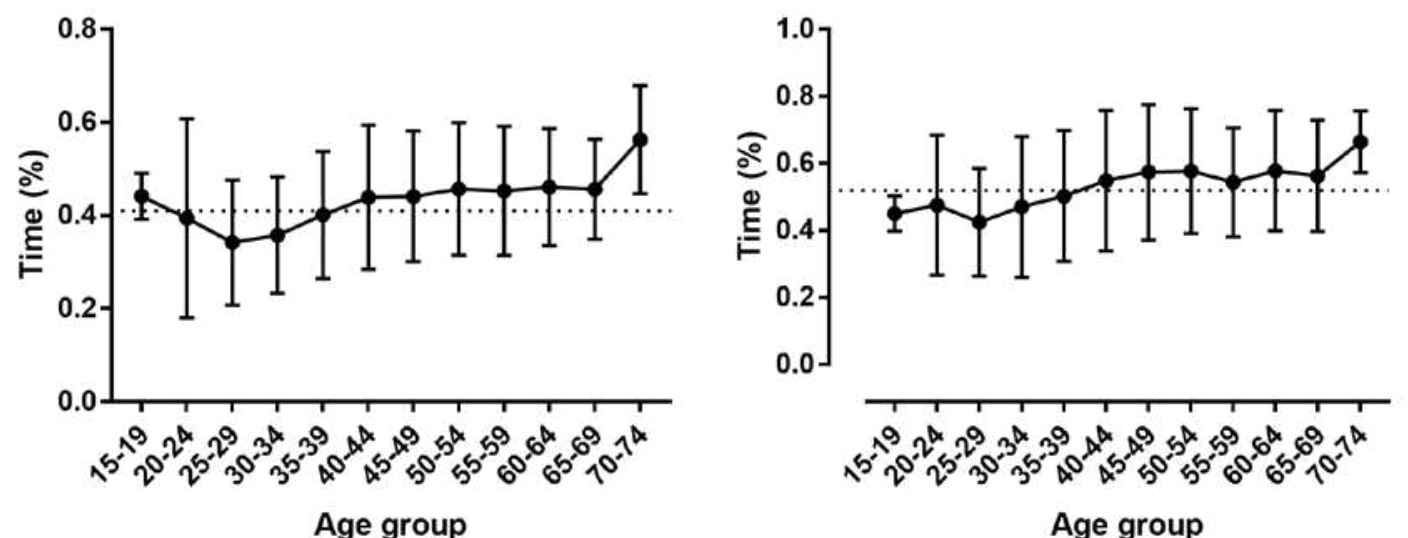

$501 \quad$ Figure 2

Age group 